\title{
Parametric excitation of slow magnetoacoustic waves in the solar corona due to photospheric periodic motions
}

\author{
T. V. Zaqarashvili, ${ }^{1}$ R. Oliver ${ }^{2}$, and J. L. Ballester ${ }^{2}$ \\ 1 Abastumani Astrophysical Observatory, Al. Kazbegi ave. 2a, 380060 Tbilisi, Georgia \\ e-mail: temury@mcs.st-and.ac.uk \\ 2 Departament de Física, Universitat de les Illes Balears, 07122 Palma de Mallorca, Spain \\ e-mail: [ramon.oliver;dfsjlbo]@uib.es
}

Received 20 July 2004 / Accepted 20 November 2004

\begin{abstract}
We suggest that the periodic shaking of coronal magnetic field lines due to photospheric periodic motions may induce the parametric excitation of slow magnetoacoustic waves in short coronal magnetic structures (with length $<50-70 \mathrm{Mm}$ ). Considering the periodic shaking of field lines as an external transversal periodic action on the coronal plasma we show that the temporal behaviour of slow wave spatial Fourier harmonics in the low plasma $\beta$ limit is governed by the Mathieu equation. Consequently, harmonics of slow magnetoacoustic waves with half the frequency of the photospheric driver have an exponential growth in time. The growth rate of slow waves is proportional to the amplitude of photospheric motions and to the value of the plasma $\beta$ in the corona. The mechanism may explain the existence of slow magnetoacoustic waves at least in short coronal loop systems (Nightingale et al. 1999, Sol. Phys., 190, 249).
\end{abstract}

Key words. magnetohydrodynamics (MHD) - Sun: corona - Sun: oscillations

\section{Introduction}

Recent space-based missions (SOHO, TRACE) have uncovered a rich spectrum of magnetohydrodynamic (MHD) oscillations in the solar corona. Two different kinds of oscillations have been frequently observed: transversal, periodic displacements of coronal loop tops and periodic intensity variations. Transversal oscillations have been understood in terms of the coronal loop global kink mode driven by a flare or a transient event (Aschwanden et al. 1999; Nakariakov et al. 1999), while intensity variations have been associated with either propagating (Deforest \& Gurman 1998; Ofman et al. 1999; Nightingale et al. 1999; De Moortel et al. 2002) or standing (Wang et al. 2003; Wang 2004) slow magnetoacoustic waves.

It is widely believed that the energy supporting the corona at a high temperature is transported from the denser and more dynamic photosphere. The mechanical energy of photospheric motions may be guided into the corona by structured magnetic fields in the form of MHD waves or electric currents (i.e. by twisting of magnetic field lines, which stores magnetic energy). Then, the energy can be released in the corona due to wave damping or magnetic reconnection.

Observational evidence of MHD waves in the corona is of scientific importance for two main reasons: first, waves may provide a clue to the long-standing coronal heating problem and, second, they may be used for the estimation of coronal parameters, i.e. for coronal seismology. However, the existence of MHD waves in the corona requires further explanation: either they penetrate from the photosphere or they are generated in situ by some mechanism. Very often waves have been observed after solar flares or some kind of energetic event. Nevertheless, observations also show wave propagation when none such events takes place in the corona, which indicates that waves in the corona may also have a source other than flares.

In this regard, the observation of slow magnetoacoustic waves in the corona is rather puzzling, as waves propagating from the photosphere must be reflected back at the transition region due to the sharp temperature gradient. Therefore, slow waves observed in the quiet corona (i.e. in the absence of any energetic events) must be excited in situ by some mechanism. While the model of slow magnetoacoustic wave propagation in the solar corona is well studied (e.g. Nakariakov et al. 2000), the source of their excitation is still unclear.

Here we suggest that periodic footpoint motions in the photosphere may resonantly amplify slow magnetoacoustic waves in the corona, at least in small loop systems. The footpoint motions of coronal magnetic structures caused by a photospheric periodic driving give rise to a periodic transversal shaking of magnetic field lines in the corona when the spatial scale of the closed magnetic system is less than Alfvénic (i.e. less than the wavelength of Alfvén waves with the period of driver). In such a system, photospheric motions will cause periodic 


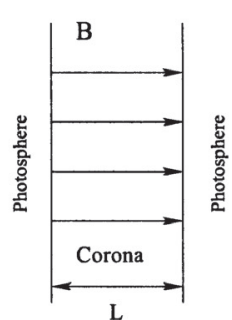

a)

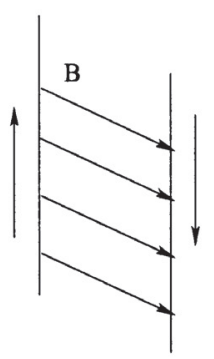

b)

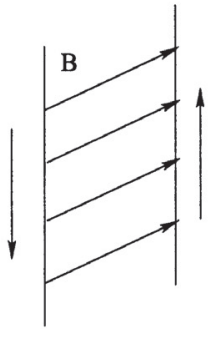

c)

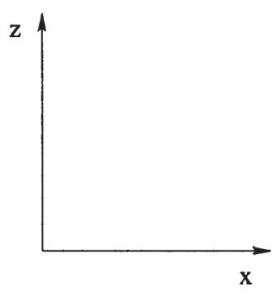

Fig. 1. Schematic picture of a coronal magnetic field tied in the dense photosphere (panel a)). The $x$-axis of a Cartesian frame is directed in the vertical direction from the photosphere. Photospheric periodic motions set up periodic oscillations of the magnetic field $z$-component when the length of the magnetic field lines in the corona, $L$, is less than the Alfvén spatial scale (panels b) and c)).

transversal oscillations of the coronal magnetic field instead of setting up a standing Alfvén wave pattern. Considering the transversal oscillation as an external action on the local coronal plasma, we show that the temporal evolution of slow wave spatial harmonics is approximately governed by the Mathieu equation (see also Zaqarashvili et al. 2002, hereafter Paper I). Thus, transversal oscillations may resonantly amplify those harmonics of slow magnetoacoustic waves with half the frequency of the photospheric driver.

The paper is organized as follows: in Sect. 2 the description of the basic state and the governing equations are detailed; in Sects. 3 and 4 an analytical and some numerical solutions are provided; in Sect. 5 the results are summarized and the phenomenon is discussed.

\section{Governing equations}

We use the ideal MHD equations with an external force, $\boldsymbol{F}$, associated with the photospheric driver:

$\frac{\partial \boldsymbol{B}}{\partial t}+(\boldsymbol{V} \cdot \nabla) \boldsymbol{B}=(\boldsymbol{B} \cdot \nabla) \boldsymbol{V}-\boldsymbol{B} \nabla \cdot \boldsymbol{V}$

$\rho \frac{\partial \boldsymbol{V}}{\partial t}+\rho(\boldsymbol{V} \cdot \nabla) \boldsymbol{V}=-\boldsymbol{\nabla}\left[p+\frac{B^{2}}{8 \pi}\right]+\frac{(\boldsymbol{B} \cdot \nabla) \boldsymbol{B}}{4 \pi}+\boldsymbol{F}$,

$\frac{\partial \rho}{\partial t}+(\boldsymbol{V} \cdot \nabla) \rho+\rho \nabla \cdot \boldsymbol{V}=0$

where $\boldsymbol{V}$ is the velocity, $\boldsymbol{B}$ is the magnetic field, $\rho$ is the density, $p$ is the pressure. Processes are assumed to be adiabatic, so the pressure and density are connected by the relation

$p=p_{0}\left(\frac{\rho}{\rho_{0}}\right)^{\gamma}$,

where $\gamma$ is the ratio of specific heats.

\subsection{Basic state}

Various coronal magnetic structures (coronal loops, arcades, prominences) are often modelled as configurations with straight magnetic field lines whose footpoints are tied in the dense photosphere (Fig. 1a). The photosphere is dominated by continuous quasi-periodic motions across the solar surface with typical periods $\sim 5 \mathrm{~min}$ (global solar oscillations, granular motions, etc.). As the magnetic field is considered to be "frozen" in the medium, these photospheric motions can cause a continuous shaking of field lines in the corona. When the period of the photospheric driver matches the period of the Alfvén eigenmodes of a closed system with spatial size $L$, then standing Alfvén waves can be excited. The typical Alfvén speed in the corona is of the order of $1000 \mathrm{~km} \mathrm{~s}^{-1}$, so photospheric motions with an $\sim 5$ min period may set up standing Alfvén waves if $L$ is of the order of or greater than $300 \mathrm{Mm}$. However, when the length of the coronal magnetic structure is considerably shorter (there are many such systems in SOHO and TRACE images), say with $L<50-70 \mathrm{Mm}$, then the photospheric motions cause a periodic shaking of magnetic field lines instead of setting up standing waves. This periodic shaking can be modelled as a spatially homogeneous oscillation of the magnetic field (see Figs. 1b, c).

Let the background medium and the magnetic field be homogeneous. Then, in a Cartesian frame with the $x$-axis directed vertically from the photosphere, the background magnetic field can be expressed as (Fig. 1a)

$B_{x}=$ const.

Now, the magnetic field $z$-component driven by periodic photospheric motions is (see Figs. 1b, c)

$B_{z}=-\alpha B_{x} \cos \left(\omega_{0} t\right)$

where $\omega_{0}$ is the frequency of photospheric motions and $\alpha$ is the relative amplitude. The corresponding velocity field is given by the induction equation as

$V_{z}=\alpha \omega_{0} \sin \left(\omega_{0} t\right) x$.

The photospheric periodic motions that drive the velocity field Eq. (7), and consequently the transversal oscillations of the coronal magnetic field, may be considered as the result of an external, periodic transversal force $\boldsymbol{F}$, acting on the plasma, expressed by

$F_{z}=\alpha \rho \omega_{0}^{2} \cos \left(\omega_{0} t\right) x$. 
The oscillations set up by the force Eq. (8) are transversal, so they do not directly cause density variations. Nevertheless, considering expressions (6)-(7) as external oscillations, one can study their parametric influence on the local compressible dynamics of the plasma. In order to study this phenomenon, the stability analysis of the state expressed by Eqs. (6)-(7) can be carried out. This means that one should consider Eqs. (6) and (7) as the basic state and then look at its stability using perturbation theory. However, it is clear that Alfvén and fast magnetoacoustic waves must be ruled out from the consideration as the size of the system is considered to be less than the Alfvén spatial scale. Fast wave perturbations propagating with a large angle to the magnetic field (i.e. fast waves propagating almost along the $z$-axis in Fig. 1) may be taken into account, but this is out of scope of this paper. Thus we consider the stability of the state expressed by Eqs. (6) and (7) to slow magnetoacoustic wave perturbations, which is justified since these perturbations have a spatial scale shorter than that of Alfvén waves due to the small plasma $\beta\left(=8 \pi p / B^{2}\right)$ in the corona.

\subsection{Perturbed equations}

We use the linear perturbation theory about the basic state described by expressions (5)-(7). Then, Eqs. (1)-(3) for the perturbations can be rewritten as

$$
\begin{aligned}
{\left[\frac{\partial}{\partial t}+V_{0} \cdot \nabla\right] \boldsymbol{b}^{\prime}=} & \left(\boldsymbol{B}_{0} \cdot \nabla\right) \boldsymbol{u}^{\prime}-\boldsymbol{B}_{0} \nabla \cdot \boldsymbol{u}^{\prime}+\left(\boldsymbol{b}^{\prime} \cdot \nabla\right) \boldsymbol{V}_{0}, \\
{\left[\frac{\partial}{\partial t}+\boldsymbol{V}_{0} \cdot \nabla\right] \boldsymbol{u}^{\prime}=} & -\frac{c_{\mathrm{s}}^{2}}{\rho_{0}} \nabla \rho^{\prime}-\frac{\nabla\left(\boldsymbol{B}_{0} \cdot \boldsymbol{b}^{\prime}\right)}{4 \pi \rho_{0}} \\
& +\frac{\left(\boldsymbol{B}_{0} \cdot \nabla\right) \boldsymbol{b}^{\prime}}{4 \pi \rho_{0}}-\left(\boldsymbol{u}^{\prime} \cdot \nabla\right) \boldsymbol{V}_{0},
\end{aligned}
$$

$\left[\frac{\partial}{\partial t}+\boldsymbol{V}_{0} \cdot \nabla\right] \rho^{\prime}=-\rho_{0} \nabla \cdot \boldsymbol{u}^{\prime}$

where $\boldsymbol{b}^{\prime}, \boldsymbol{u}^{\prime}$ and $\rho^{\prime}$ are the perturbations of the magnetic field, velocity and density, respectively. Moreover, $\boldsymbol{B}_{0}=$ $\left(B_{x}, 0, B_{z}(t)\right), V_{0}=\left(0,0, V_{z}(t, x)\right)$ and $\rho_{0}$ are the equilibrium magnetic field, velocity and density, while $c_{\mathrm{s}}=\sqrt{\gamma p_{0} / \rho_{0}}$ is the adiabatic sound speed.

It must be noticed here that the expression of the external driver, $\boldsymbol{F}$, is not directly included in the perturbed Eqs. (9)-(11), which points to its intrinsically parametric influence. This means that photospheric motions do not directly influence the compressible dynamics of the corona. Instead, they may act on the coronal plasma by setting up periodic shearing motions and oscillating magnetic field component of Eqs. (7) and (6), respectively. The terms with $\boldsymbol{V}_{0}$ or $\boldsymbol{B}_{0}$ in Eqs. (9)-(11) have a clear parametric origin as they also include the perturbations. In the case of a direct action the equations must be inhomogeneous and at least one term must be of external origin only, i.e. it must include only $\boldsymbol{V}_{0}$ or $\boldsymbol{B}_{0}$ not multiplied by perturbed quantities.

For simplicity, we consider only the two-dimensional case, in which slow magnetoacoustic waves are polarised in the plane of transversal oscillations, i.e. in the $X o Z$ plane. The consideration of the third dimension is not important for the process, so it can be neglected. Then, using expressions (6) and (7) we get

$$
\begin{aligned}
& {\left[\frac{\partial}{\partial t}+V_{z} \frac{\partial}{\partial z}\right] b_{z}^{\prime}=B_{x} \frac{\partial u_{z}^{\prime}}{\partial x}-B_{z} \frac{\partial u_{x}^{\prime}}{\partial x}+V_{z x} b_{x}^{\prime}} \\
& {\left[\frac{\partial}{\partial t}+V_{z} \frac{\partial}{\partial z}\right] b_{x}^{\prime}=B_{z} \frac{\partial u_{x}^{\prime}}{\partial z}-B_{x} \frac{\partial u_{z}^{\prime}}{\partial z}} \\
& {\left[\frac{\partial}{z}+V_{z} \frac{\partial}{\partial z}\right] u_{z}^{\prime}=-\frac{c_{\mathrm{s}}^{2}}{\rho_{0}} \frac{\partial \rho^{\prime}}{\partial z}+\frac{B_{x}}{4 \pi \rho_{0}} \frac{\partial b_{z}^{\prime}}{\partial x}} \\
& {\left[\frac{\partial}{\partial t}+V_{z} \frac{\partial}{\partial z}\right] u_{x}^{\prime}=-\frac{B_{\mathrm{s}}^{2}}{\rho_{0}} \frac{\partial \rho_{0}^{\prime}}{\partial x}+\frac{B_{z}^{\prime}}{\partial \pi \rho_{0}} \frac{\partial b_{x}^{\prime}}{\partial z}-\frac{B_{z}}{4 \pi \rho_{0}} \frac{\partial b_{z}^{\prime}}{\partial x}} \\
& {\left[\frac{\partial}{\partial t}+V_{z} \frac{\partial}{\partial z}\right] \rho^{\prime}=-\rho_{0}\left[\frac{\partial u_{z}^{\prime}}{\partial z}+\frac{\partial u_{x}^{\prime}}{\partial x}\right]}
\end{aligned}
$$

where

$V_{z x}=\frac{\partial V_{z}}{\partial x}=\alpha \omega_{0} \sin \left(\omega_{0} t\right)$

is the gradient of the shearing motion given by Eq. (7).

Equations (12)-(16) include both spatial and temporal inhomogeneities due to the shearing motion (7), which does not allow us to perform a Fourier analysis. Fortunately, the spatial inhomogeneity in the linearized MHD equations due to a linear shear flow vanishes when a comoving frame is used (Kelvin 1887; Goldreich \& Lynden-Bell 1965; Marcus \& Press 1977; Craik \& Allen 1992; Balbus \& Hawley 1992; Chagelishvili et al. 1993; Zaqarashvili 2000, and Paper I). Consequently, the wavevector of perturbations undergoes a temporal drift in phase space. Then, straightforward calculations using Fourier analysis with a time periodic wavenumber (see Paper I, for details) leads to the following equations with only time-dependent coefficients,

$\frac{\mathrm{d} b_{x}}{\mathrm{~d} t}=i k_{z} B_{z} u_{x}-i k_{z} B_{x} u_{z}$,

$\frac{\mathrm{d} u_{z}}{\mathrm{~d} t}=-i k_{z} \frac{c_{\mathrm{s}}^{2}}{\rho_{0}} \rho-\frac{i B_{x}}{4 \pi \rho_{0} k_{z}}\left[k_{z}^{2}+K_{x}^{2}\right] b_{x}-V_{z x} u_{x}$,

$\frac{\mathrm{d} u_{x}}{\mathrm{~d} t}=-i K_{x} \frac{c_{\mathrm{s}}^{2}}{\rho_{0}} \rho+\frac{i B_{z}}{4 \pi \rho_{0} k_{z}}\left[k_{z}^{2}+K_{x}^{2}\right] b_{x}$,

$\frac{\mathrm{d} \rho}{\mathrm{d} t}=-i \rho_{0}\left(k_{z} u_{z}+K_{x} u_{x}\right)$,

where $b_{x}, u_{x}, u_{z}$ and $\rho$ are the amplitudes of the spatial Fourier harmonics, whereas $k_{z}$ and

$K_{x}(t)=k_{x}+\alpha k_{z} \cos \left(\omega_{0} t\right)$

are the wavenumbers. Note that the periodic component in $K_{x}$ comes from the periodic shearing motion, Eq. (7).

In general, these equations include the solutions of both fast and slow magnetoacoustic waves, the influence of the transversal oscillations being expressed by the terms with the coefficient $\alpha$. Neglecting the external action (i.e. taking $\alpha=0$ ) leads 
to the usual differential equations for magnetoacoustic waves. But, as already noticed above, we only consider slow magnetoacoustic waves as their wavelength can be shorter than $L$ due to the small plasma $\beta$ in the corona.

We begin our study of the differential Eqs. (18)-(21) by looking for approximate analytical solutions for slow magnetoacoustic waves. Then, numerical simulations will be used to test the analytical solutions and to confirm the insight gained from them. In this paper we are only concerned with the low plasma $\beta$ approximation, i.e. $\beta \ll 1$, which is reasonable in the short coronal magnetic structures (with length $<50-70 \mathrm{Mm}$ ) considered here. This can be confirmed following Gary (2001): since photospheric motions (for instance, buffeting by granulation) are assumed to shake the loop, its footpoints must be located away from the most internal part of sunpot umbrae, i.e. in regions of moderate magnetic fields. On the other hand, if we assume that the shape of the loop is semicircular, its height is around $20 \mathrm{Mm}$. Then, from Fig. 3 in Gary (2001) our short loops have $\beta \approx 0.1$ along all their length.

\section{Analytical study}

A simple algebraic transformation of Eqs. (18)-(21) leads to two second-order differential equations,

$$
\begin{aligned}
\frac{\mathrm{d}^{2} b_{x}}{\mathrm{~d} t^{2}}-\frac{2 k_{z} V_{z x}}{k_{x}} & \frac{\mathrm{d} b_{x}}{\mathrm{~d} t}+\frac{B_{z}^{2}+B_{x}^{2}}{4 \pi \rho_{0}}\left(k_{z}^{2}+K_{x}^{2}\right) b_{x}= \\
& \frac{k_{z} c_{\mathrm{s}}^{2}\left(B_{z} K_{x}-B_{x} k_{z}\right)}{\rho_{0}} \rho-\frac{2 k_{z} V_{z x} B_{x}}{k_{x} \rho_{0}} \frac{\mathrm{d} \rho}{\mathrm{d} t},
\end{aligned}
$$

$$
\begin{aligned}
\frac{\mathrm{d}^{2} \rho}{\mathrm{d} t^{2}}+\frac{2 k_{z} V_{z x}}{k_{x}} & \frac{\mathrm{d} \rho}{\mathrm{d} t}+c_{\mathrm{s}}^{2}\left(k_{z}^{2}+K_{x}^{2}\right) \rho= \\
& \frac{B_{z} K_{x}-B_{x} k_{z}}{4 \pi k_{z}}\left(k_{z}^{2}+K_{x}^{2}\right) b_{x}+\frac{2 k_{z} V_{z x} \rho_{0}}{k_{x} B_{x}} \frac{\mathrm{d} b_{x}}{\mathrm{~d} t} .
\end{aligned}
$$

These are the coupled expressions governing the propagation of fast and slow magnetoacoustic waves in the presence of external transversal oscillations. Finding an analytical solution to these equations is a complicated task, although it is possible to determine the main properties of the wave dynamics by choosing some appropriate approximations.

One may ignore the magnetic field effects and consider only Eq. (24) without the right hand-side terms,

$$
\frac{\mathrm{d}^{2} \rho}{\mathrm{d} t^{2}}+\frac{2 k_{z} V_{z x}}{k_{x}} \frac{\mathrm{d} \rho}{\mathrm{d} t}+c_{\mathrm{s}}^{2}\left(k_{z}^{2}+K_{x}^{2}\right) \rho=0 .
$$

This equation describes sound waves in the presence of the periodic shearing motion of Eq. (7). It can be easily shown that this expression leads to the Mathieu equation and that, consequently, the solution is exponentially growing, which means that periodic hydrodynamic shear flows are unstable (Craik \& Allen 1992). Nevertheless, the presence of the magnetic field may stabilize the state. Hence, in order to study the influence of the magnetic field on the hydrodynamic instability of this periodic shearing flow one should solve both Eqs. (23) and (24). To derive an analytical solution two approximations are made: the low plasma $\beta$ limit (such as already mentioned) and weak external forcing, i.e. $\alpha \ll 1$.
For slow magnetoacoustic waves in a low plasma $\beta$ (i.e. $c_{\mathrm{s}} \ll V_{\mathrm{A}}$, where $V_{\mathrm{A}}=B_{x} / \sqrt{4 \pi \rho_{0}}$ is Alfvén speed) the following relation holds, $b_{x} / B_{x} \ll \rho / \rho_{0}$ (such as can be easily derived from Eq. (23) with $\alpha=0$ ). Therefore, the terms with the first derivative of $b_{x}$ (which also include the small parameter $\alpha$ ) are smaller than the rest in Eqs. (23)-(24) and can be neglected. After substituting the second derivative of $b_{x}$ by the square of the slow magnetoacoustic wave frequency, $\omega_{\mathrm{s}}$, multiplied by $b_{x}$ (by this substitution we exclude fast waves from our analysis) and neglecting the terms with $\alpha^{2}$, Eqs. (23)-(24) can be rewritten as

$$
\begin{gathered}
{\left[-\omega_{\mathrm{s}}^{2}+V_{\mathrm{A}}^{2}\left(k_{z}^{2}+K_{x}^{2}\right)\right] b_{x}=\frac{k_{z} c_{\mathrm{s}}^{2}\left(B_{z} k_{x}-B_{x} k_{z}\right)}{\rho_{0}} \rho} \\
-\frac{2 k_{z} V_{z x} B_{x}}{k_{x} \rho_{0}} \frac{\mathrm{d} \rho}{\mathrm{d} t},
\end{gathered}
$$

$\frac{\mathrm{d}^{2} \rho}{\mathrm{d} t^{2}}+\frac{2 k_{z} V_{z x}}{k_{x}} \frac{\mathrm{d} \rho}{\mathrm{d} t}+c_{\mathrm{s}}^{2}\left(k_{z}^{2}+K_{x}^{2}\right) \rho=$

$$
\frac{B_{z} k_{x}-B_{x} k_{z}}{4 \pi k_{z}}\left(k_{z}^{2}+K_{x}^{2}\right) b_{x}
$$

As we will see below, numerical simulations of Eqs. (18)-(21) (from which Eqs. (26) and (27) originate) reveal that this approximation correctly describes the slow wave dynamics.

Substitution of $b_{x}$ from Eq. (27) into Eq. (26) leads to

$$
\begin{aligned}
\frac{\mathrm{d}^{2} \rho}{\mathrm{d} t^{2}}+\frac{2 k_{z} V_{z x}}{k_{x}} \frac{\mathrm{d} \rho}{\mathrm{d} t}+c_{\mathrm{s}}^{2}\left(k_{z}^{2}+K_{x}^{2}\right) \rho= \\
\\
\frac{\left(B_{z} k_{x}-B_{x} k_{z}\right)^{2} c_{\mathrm{s}}^{2}}{4 \pi \rho_{0}\left[V_{\mathrm{A}}^{2}\left(k_{z}^{2}+K_{x}^{2}\right)-\omega_{\mathrm{s}}^{2}\right]}\left(k_{z}^{2}+K_{x}^{2}\right) \rho \\
-\frac{2 V_{z x} B_{x}\left(B_{z} k_{x}-B_{x} k_{z}\right)\left(k_{z}^{2}+K_{x}^{2}\right)}{4 \pi \rho_{0} k_{x}\left[V_{\mathrm{A}}^{2}\left(k_{z}^{2}+K_{x}^{2}\right)-\omega_{\mathrm{s}}^{2}\right]} \frac{\mathrm{d} \rho}{\mathrm{d} t} .
\end{aligned}
$$

This equation can be simplified by again neglecting the terms with $\alpha^{2}$, so we get

$$
\begin{aligned}
\frac{\mathrm{d}^{2} \rho}{\mathrm{d} t^{2}}-\frac{2 k_{z} V_{z x}}{k_{x}} & \frac{\omega_{\mathrm{s}}^{2}}{V_{\mathrm{A}}^{2}\left(k_{z}^{2}+K_{x}^{2}\right)-\omega_{\mathrm{s}}^{2}} \frac{\mathrm{d} \rho}{\mathrm{d} t} \\
& +c_{\mathrm{s}}^{2}\left(k_{z}^{2}+K_{x}^{2}\right)\left[\frac{k_{x}^{2} V_{\mathrm{A}}^{2}-\omega_{\mathrm{s}}^{2}}{V_{\mathrm{A}}^{2}\left(k_{z}^{2}+K_{x}^{2}\right)-\omega_{\mathrm{s}}^{2}}\right] \rho=0 .
\end{aligned}
$$

This is the Hill equation and can be easily transformed into the Mathieu equation with the substitution

$\rho=\rho_{1}(t) \exp \left\{\int\left[\frac{k_{z} V_{z x}}{k_{x}} \frac{\omega_{\mathrm{s}}^{2}}{V_{\mathrm{A}}^{2}\left(k_{z}^{2}+K_{x}^{2}\right)-\omega_{\mathrm{s}}^{2}}\right] \mathrm{d} t\right\}$,

so we have

$$
\begin{aligned}
\frac{\mathrm{d}^{2} \rho_{1}}{\mathrm{~d} t^{2}}+\left[c_{\mathrm{s}}^{2}\left(k_{z}^{2}+K_{x}^{2}\right)\right. & \frac{k_{x}^{2} V_{\mathrm{A}}^{2}-\omega_{\mathrm{s}}^{2}}{V_{\mathrm{A}}^{2}\left(k_{z}^{2}+K_{x}^{2}\right)-\omega_{\mathrm{s}}^{2}} \\
& \left.+\frac{\alpha k_{z}}{k_{x}} \frac{\omega_{0}^{2} \omega_{\mathrm{s}}^{2} \cos \left(\omega_{0} t\right)}{V_{\mathrm{A}}^{2}\left(k_{z}^{2}+K_{x}^{2}\right)-\omega_{\mathrm{s}}^{2}}\right] \rho_{1}=0
\end{aligned}
$$


Taking into account the time dependence of $K_{x}$ we get

$$
\begin{aligned}
\frac{\mathrm{d}^{2} \rho_{1}}{\mathrm{~d} t^{2}}+\left[\Omega^{2}+\right. & \frac{\alpha \omega_{\mathrm{s}}^{2}}{V_{\mathrm{A}}^{2}\left(k_{z}^{2}+k_{x}^{2}\right)-\omega_{\mathrm{s}}^{2}} \\
& \left.\times\left(\frac{k_{z}}{k_{x}} \omega_{0}^{2}-\frac{2 k_{x} k_{z} \Omega^{2}}{k_{z}^{2}+k_{x}^{2}}\right) \cos \left(\omega_{0} t\right)\right] \rho_{1}=0,
\end{aligned}
$$

where

$\Omega^{2}=\frac{c_{\mathrm{s}}^{2}\left(k_{z}^{2}+k_{x}^{2}\right)\left(k_{x}^{2} V_{\mathrm{A}}^{2}-\omega_{\mathrm{s}}^{2}\right)}{V_{\mathrm{A}}^{2}\left(k_{z}^{2}+k_{x}^{2}\right)-\omega_{\mathrm{s}}^{2}}$.

Note that when $\alpha=0$ this equation leads exactly to the usual expression for slow magnetoacoustic waves as $\Omega^{2}$ is the square of the slow wave frequency, $\omega_{\mathrm{s}}^{2}$; one can easily recover the dispersion relation for slow magnetoacoustic waves by substituting $\Omega^{2}$ by $\omega_{\mathrm{s}}^{2}$ in expression (32).

So we finally obtain

$$
\frac{\mathrm{d}^{2} \rho_{1}}{\mathrm{~d} t^{2}}+\left[\omega_{\mathrm{s}}^{2}+\delta \cos \left(\omega_{0} t\right)\right] \rho_{1}=0
$$

where

$\delta=\frac{\alpha \omega_{\mathrm{s}}^{2}}{V_{\mathrm{A}}^{2}\left(k_{z}^{2}+k_{x}^{2}\right)-\omega_{\mathrm{s}}^{2}}\left(\frac{k_{z}}{k_{x}} \omega_{0}^{2}-\frac{2 k_{x} k_{z} \omega_{\mathrm{s}}^{2}}{k_{z}^{2}+k_{x}^{2}}\right)$.

Equation (33) is the classical Mathieu equation and the main resonance occurs when (Landau \& Lifshitz 1988)

$\omega_{\mathrm{s}}^{2} \approx \frac{\omega_{0}^{2}}{4}$.

Therefore, the harmonics of slow magnetoacoustic waves with half the frequency of transversal oscillations grow exponentially in time. The resonant solution of Eq. (33) is

$\rho_{1}(t)=\rho_{1}(0) \exp \left(\frac{|\delta|}{2 \omega_{0}} t\right)\left(\cos \frac{\omega_{0}}{2} t-\sin \frac{\omega_{0}}{2} t\right)$.

Taking into account the relation (35) and the approximate expression $\omega_{\mathrm{s}} \approx c_{\mathrm{s}} k_{x}$ for the slow wave frequency, we get for the growth rate

$$
\frac{|\delta|}{2 \omega_{0}} \approx \frac{\alpha \omega_{0}}{4} \frac{c_{\mathrm{s}}^{2}}{V_{\mathrm{A}}^{2}} \frac{\left|k_{x}\right|\left|k_{z}\right|}{k_{x}^{2}+k_{z}^{2}} \frac{k_{x}^{2}+2 k_{z}^{2}}{k_{x}^{2}+k_{z}^{2}} .
$$

This formula indicates that the maximum value of the growth rate takes place for $\left|k_{z}\right| /\left|k_{x}\right| \approx 1.3$; hence, the energy transfer is maximum for slow magnetoacoustic waves which propagate with an angle of $\sim 50^{\circ}$ with respect to the equilibrium magnetic field. The analytical solution also predicts that the growth rate of slow magnetoacoustic waves varies linearly with the amplitude of transversal oscillations, $\alpha$, and the plasma $\beta$,

$$
\frac{|\delta|}{2 \omega_{0}} \sim \alpha \beta
$$

Thus, the energy transmission from transversal oscillations to slow magnetoacoustic waves is more efficient in a medium with high $\beta$ (with $\beta$ still smaller than 1 ). Finally, these solutions have a resonant interval given by

$\left|\omega_{\mathrm{s}}-\frac{\omega_{0}}{2}\right|<\left|\frac{\delta}{\omega_{0}}\right|$
As a consequence, a strong external action generates a wide range of resonant slow magnetoacoustic wave harmonics in plasmas with moderately small $\beta$.

Thus, the periodic shearing motion Eq. (7) is unstable even in the presence of a magnetic field. The difference between hydrodynamical instabilities (expressed by Eq. (25)) and MHD instabilities (expressed by Eq. (28)) is that the growth rate of resonant slow magnetoacoustic waves depends on the plasma $\beta$ and the amplitude of the external forcing $\alpha$ (Eq. (38)), while the growth rate of the hydrodynamic resonant solution depends only on $\alpha$.

\section{Numerical simulations}

The above analytical solutions are useful in the limit of very small plasma $\beta$ for "weak" transversal oscillations (i.e. for $\alpha$ not too large). Nevertheless, to obtain solutions to Eqs. (18)-(21), from which the expressions considered in Sect. 3 originate, for any values of $\beta$ and $\alpha$ it is necessary to resort to numerical techniques. Solving this set of ordinary differential equations is straightforward and it is only necessary to supplement it with the appropriate initial conditions for $b_{x}, u_{x}, u_{z}$ and $\rho$. Since we are interested in the energy transfer from the external forcing to the slow magnetoacoustic modes of the medium, to derive those initial conditions we simply consider that the wave is propagating in the system at $t=0$, when the transversal oscillation is "turned on". The initial values of the four physical variables can be determined after taking into account that slow modes propagating in the present infinite, uniform medium satisfy Eqs. (18)-(21) without the forcing term $V_{z x} u_{x}$, that vanishes for $t=0$, and with $B_{z}^{0} \equiv B_{z}(0)=-\alpha B_{x}$ and $K_{x}^{0} \equiv K_{x}(0)=k_{x}+\alpha k_{z}$. Then, using a temporal dependence of the form $\exp (i \omega t)$, the amplitudes $b_{x}^{0}, u_{x}^{0}, u_{z}^{0}$ and $\rho^{0}$ (i.e. the initial conditions) of the physical variables satisfy the equations

$$
i \omega b_{x}^{0}=i k_{z} B_{z}^{0} u_{x}^{0}-i k_{z} B_{x} u_{z}^{0}
$$

$i \omega u_{x}^{0}=-i K_{x}^{0} c_{\mathrm{s}}^{2} \frac{\rho^{0}}{\rho_{0}}+i \frac{B_{z}^{0}}{4 \pi \rho_{0}} \frac{k_{z}^{2}+K_{x}^{0^{2}}}{k_{z}} b_{x}^{0}$,

$$
i \omega u_{z}^{0}=-i k_{z} c_{\mathrm{s}}^{2} \frac{\rho^{0}}{\rho_{0}}-i \frac{B_{x}}{4 \pi \rho_{0}} \frac{k_{z}^{2}+K_{x}^{0^{2}}}{k_{z}} b_{x}^{0},
$$

$$
i \omega \rho^{0}=-i \rho_{0}\left(K_{x}^{0} u_{x}^{0}+k_{z} u_{z}^{0}\right)
$$

These expressions constitute a homogeneous set of algebraic equations whose determinant must be zero to have a non-trivial solution, a condition that yields the dispersion relation of magnetoacoustic waves.

Now, the numerical procedure is started by first imposing the frequency and the $x$ - and $z$-components of the wavenumber of a slow mode, bearing in mind that $\omega, k_{x}$ and $k_{z}$ must satisfy the dispersion relation. Next, Eqs. (40)-(43) allow one to calculate the initial conditions except for an arbitrary constant, so we choose the value $\rho^{0}=1$, say, and compute $b_{x}^{0}, u_{x}^{0}$ and $u_{z}^{0}$, from three of the above four equations. Then, the numerical integration of the differential Eqs. (18)-(21) can be carried out. 


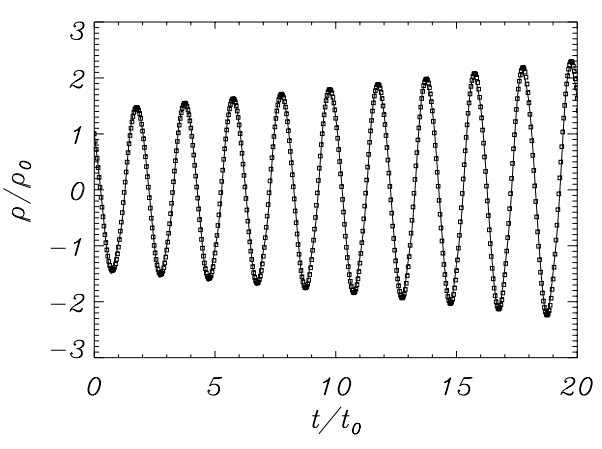

Fig. 2. Comparison between the numerical solution of Eqs. (18)-(21) and the analytical solution, given by Eqs. (29), (36) and (37). The dimensionless density perturbation, $\rho / \rho_{0}$, is plotted as a function of dimensionless time, $t / t_{0}$, with $t_{0}=2 \pi / \omega_{0}$ the period of the transversal oscillations. Solid line: numerical solution, squares: analytical solution.

Our first task is to check that the numerical solutions are correct by comparing them with the analytical formulas derived in the previous section. It must be stressed again that, for given values of $\alpha$ and $\beta$, not all slow wave harmonics are resonantly amplified and that only those that satisfy the resonant condition (35), i.e. those with the right wavenumber, are able to extract energy from the transversal oscillations. Numerical solutions outside the resonant interval given by Eq. (39) show no signs of amplification and it is necessary to "tune" the wavenumber components, $k_{x}$ and $k_{z}$, until a resonant solution is obtained. For a detailed discussion of the transition from nonresonant to amplified solutions, see Paper I. Once a resonant solution is found numerically, its agreement with the analytical one is excellent, as exemplified by Fig. 2 .

The analytical approximation developed in Sect. 3 predicts a stronger amplification of waves propagating at $50^{\circ}$ from the equilibrium field and a linear dependence of the growth rate with the plasma- $\beta$. In spite of the approximations made to obtain the analytical solution, these two aspects of the resonant amplification of slow waves are found in the numerical solutions. The first of them is well illustrated in Fig. 3, in which the largest amplification is found for a propagation angle $\theta \simeq 50^{\circ}$.

Regarding the dependence of the amplification rate on the plasma $\beta$, Fig. 4 presents the numerical calculations for the amplification of slow waves propagating with $k_{x}=k_{z}$ in a plasma with three values of $\beta$. It is obvious that the instability becomes larger as the beta increases and the amplification rate of the slow wave increases almost linearly with $\beta$.

It is straightforward to check that as predicted by Eq. (35) the resonant solutions in Figs. 2-4 have a frequency equal to half the frequency of transversal oscillations, i.e. a period equal to $2 t_{0}$.

\section{Discussion}

Slow waves with no relation to energetic events have been frequently observed in the corona in the last few years (e.g. Deforest \& Gurman 1998; Ofman et al. 1999; Nightingale et al. 1999; De Moortel et al. 2002; Wang et al. 2003; Wang 2004). This paper suggests a mechanism for the generation of slow waves in the corona in the absence of flares or other energetic events. Although it is reasonable to suggest that the source of slow waves lies in the photosphere, their propagation from the solar surface is quite complicated due to the sharp temperature gradient at the transition region and the subsequent rapid increase of the phase velocity, that forces slow waves to reflect back to the chromosphere. Therefore, the photospheric source cannot be considered as a direct driver of slow waves since they cannot directly penetrate through the transition region, so that slow waves must be generated in situ in the corona by some other mechanism.

Apart from direct driving, there is another kind of external action which can effectively give rise to coronal oscillations via the so-called parametric action. In this phenomenon the external driver may amplify the oscillations through the periodic variation of a system parameter (a mechanical example of such action is an ordinary mathematical pendulum with a periodically varying length; see e.g. Zaqarashvili \& Roberts 2002). In this paper we suggest that quasi-periodic photospheric motions can excite slow magnetoacoustic waves through the periodic shaking of magnetic field lines in the corona (see Fig. 1). When the length of coronal magnetic structures (loops, arcades) is less than the Alfvén spatial scale (i.e. the wavelength of Alfvén waves with a period similar to that of photospheric motions), then the photospheric motions will cause a periodic variation of the coronal magnetic field instead of setting up a standing pattern of Alfvén waves. The corresponding velocity and magnetic field components can be written as in Eqs. (6) and (7). As the medium density is considered to be homogeneous, the instability of the state expressed by these equations due to phase mixing is absent. Nevertheless, we have found that the system is unstable in front of slow magnetoacoustic waves. It has been shown that the temporal dynamics of the slow wave spatial harmonics is approximately governed by the Mathieu equation (Eq. (33)), which points to the parametric origin of the instability. Both analytical and numerical studies indicate that the amplification is optimum for slow magnetoacoustic waves with half the frequency of the external action (see also Paper I),

$\omega_{\mathrm{s}} \sim \frac{\omega_{0}}{2}$

The growth rate of slow magnetoacoustic waves depends on the relative amplitude of the photospheric motions, $\alpha$, and on the plasma $\beta$, with a stronger amplification for high $\beta$ (note that here $\beta$ is considered to be less than 1). It has also been found that slow waves propagating with different angles about the unperturbed magnetic field are amplified, with the maximum growth rate occurring at a propagation angle $\sim 50^{\circ}$.

Thus, the periodic footpoint motion of short loops or narrow arcades (with a spatial scale smaller than 50-70 Mm) may parametrically excite slow magnetoacoustic waves in the corona. Nightingale et al. (1999) found propagating compressible disturbances in a short loop system $(\sim 30 \mathrm{Mm})$, which they interpreted as slow magnetoacoustic waves. Although these authors suggested reconnection as the source of the waves, the present mechanism is also a good candidate for their excitation. Note, also, the recent discovery of loop oscillations with a period around 10 min (Terradas et al. 2004). These intensity 

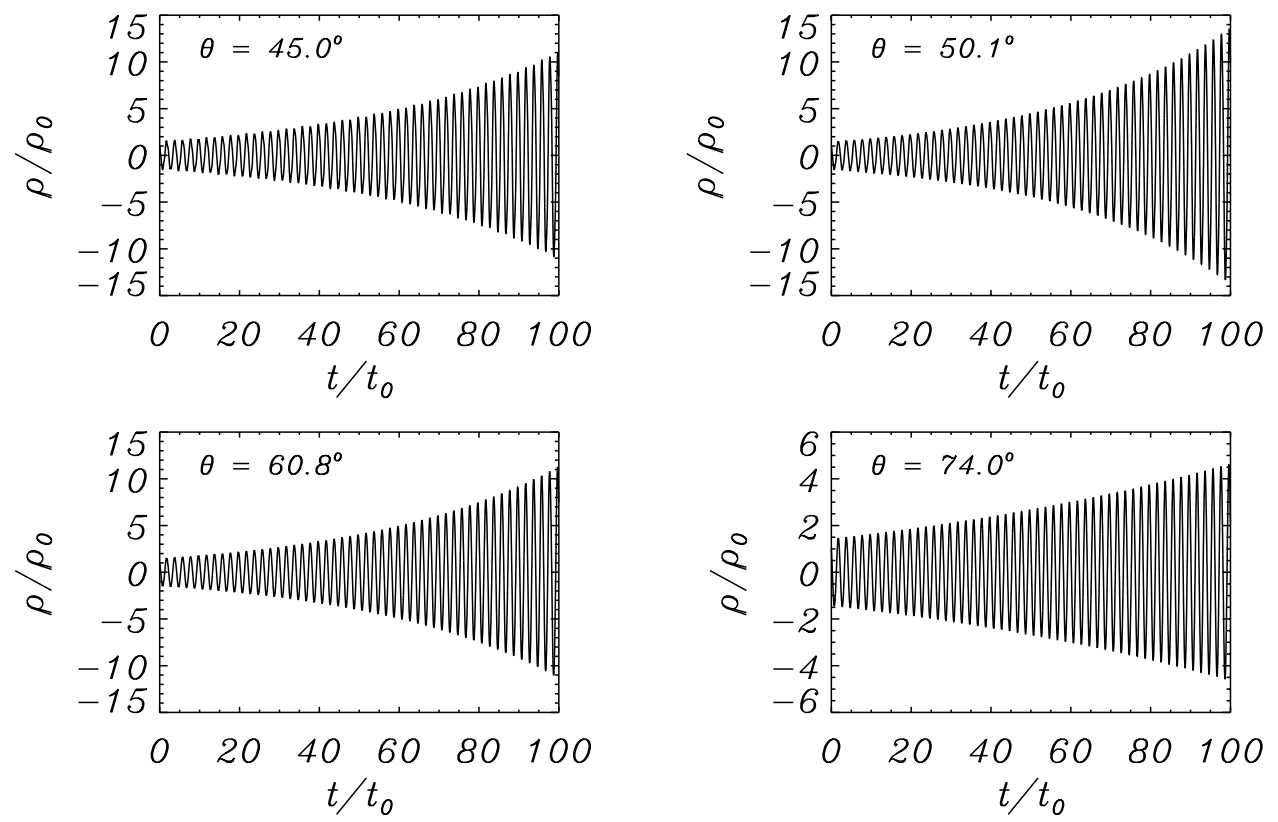

Fig. 3. Amplification of slow waves by transversal oscillations with a relative amplitude $\alpha=0.1$ in a plasma with $\beta=0.24$. Before transversal oscillations start to act at $t=0$, a slow wave propagates at an angle $\theta$ to the $x$-axis. The density perturbation is plotted for $\theta=45.0^{\circ}, \theta=50.1^{\circ}$, $\theta=60.8^{\circ}$ and $\theta=74.0^{\circ}$.

oscillations had been unnoticed in a previous analysis of the same data set, probably because of their small amplitude, and their origin is not clear. Taking into account that their period is twice that of the photospheric oscillations, we here suggest that these 10-min loop oscillations may be the result of parametric amplification by photospheric motions. Now, one may wonder why such 10-min intensity oscillations are not routinely observed in TRACE data, for example. The answer to this question can be that the efficiency of the mechanism is such that the amplitude of driven slow waves in coronal loops is quite small. In fact this is the case with the oscillations reported by Terradas et al. (2004), who found that the amplitude of this signal is near the noise level. More observations and links between photospheric and coronal events are necessary. In addition, new theoretical work is needed to estimate the energy that can be transferred by means of this parametric amplification from the photosphere to the corona.

In the low $\beta$, long-wavelength regime the dispersion laws for the slow magnetoacoustic mode and ion-sound waves (in the case of pure longitudinal propagation) become practically the same. Thus, in the case of pure longitudinal propagation the slow mode described in this paper should be better referred to as a low frequency wave in the range of slow magnetoacoustic and ion-sound waves. Nevertheless we stress once more that the resonant phenomenon investigated here takes place for propagation at a large angle with respect to the unperturbed magnetic field.

Acknowledgements. The work of T.Z. was supported by NATO Reintegration Grant FEL.RIG 980755 and a grant of the Georgian Academy of Sciences. T.Z. also acknowledges the great hospitality of the Departament de Física of the Universitat de les Illes Balears, where this work was done. R.O. and J.L.B. acknowledge the financial support received from MCyT under grant AYA2003-00123.
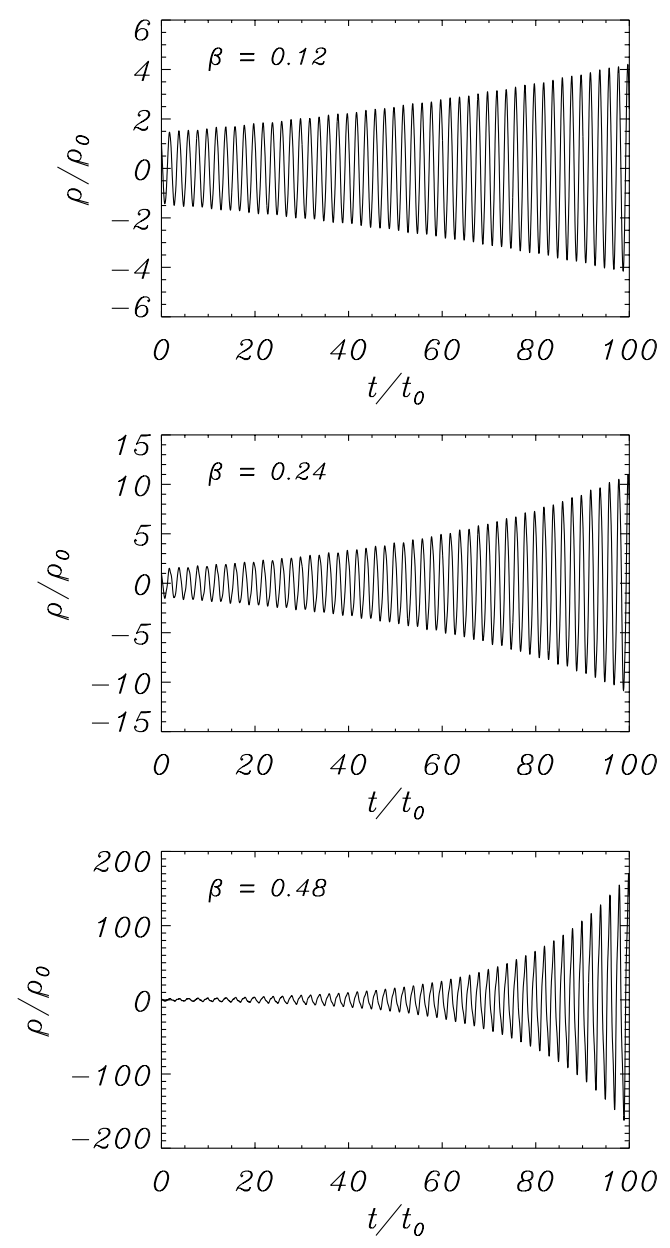

Fig. 4. Amplification of slow waves, propagating at an angle $\theta=45^{\circ}$ to the unperturbed magnetic field, by transversal oscillations with a relative amplitude $\alpha=0.1$. The density perturbation is plotted for $\beta=0.12, \beta=0.24$ and $\beta=0.48$. 


\section{References}

Aschwanden, M. J., Fletcher, L., Schrijver, C. J., \& Alexander, D. 1999, ApJ, 520, 880

Balbus, S. A., \& Hawley, J. H. 1992, ApJ, 400, 610

Chagelishvili, G. D., Hristov, T. S., Chanishvili, R. G., \& Lominadze, J. G. 1993, Phys. Rev. E, 47, 366

Craik, A. D. D., \& Allen, H. R. 1992, J. Fluid Mech., 238, 613

Deforest, C. E., \& Gurman, G. B. 1998, ApJ, 501, L217

De Moortel, I., Ireland, J., Hood, A. W., \& Walsh, R. W. 2002, A\&A, 387, L13

Gary, G. A. 2001, Sol. Phys., 203, 71

Goldreich, P., \& Lynden-Bell, D. 1965, MNRAS, 130, 125

Kelvin, W. 1887, Philos. Mag., 24, Ser. 5, 188

Landau, L. D., \& Lifshitz, E. M. 1988, Theoretical Mechanics (Moskow: Nauka)

Marcus, P., \& Press, W. H. 1977, J. Fluid Mech., 79, 525
Nakariakov, V. M., Ofman, L., Deluca, E. E., Roberts, B., \& Davila, J. M. 1999, Science, 285, 862

Nakariakov, V. M., Verwichte, E., Berghmans, D., \& Robbrecht, E. 2000, A\&A, 362, 1151

Nightingale, R. W., Aschwanden, M. J., \& Hurlburt, N. E. 1999, Sol. Phys., 190, 249

Ofman, L., Nakariakov, V. M., \& DeForest, C. E. 1999, ApJ, 514, 441

Terradas, J., Oliver, R., \& Ballester, J. L. 2004, ApJ, 614, 435

Wang, T. J. 2004, in Proc. of SOHO 13, Waves, oscillations and smallscale transient events in the solar atmosphere: a joint view from SOHO and TRACE, ESA-SP, 547, 417

Wang, T. J., Solanki, S. K., Innes, D. E., Curdt, W., \& Marsch, E. 2003, A\&A, 402, L1

Zaqarashvili, T. V. 2000, Phys. Rev. E, 62, 2745

Zaqarashvili, T. V., \& Roberts, B. 2002, Phys. Rev. E, 66, 026401

Zaqarashvili, T. V., Oliver, R., \& Ballester, J. L. 2002, ApJ, 569, 519, Paper I 